\title{
Maximizing the Energy Density of Dielectric Elastomer Generators Using Equi-Biaxial Loading
}

\section{Citation}

Huang, Jiangshui, Samuel Shian, Zhigang Suo, and David R. Clarke. Forthcoming. Maximizing the energy density of dielectric elastomer generators using equi-biaxial loading. Advanced Functional Materials.

\section{Permanent link}

http://nrs.harvard.edu/urn-3:HUL.InstRepos:10476713

\section{Terms of Use}

This article was downloaded from Harvard University's DASH repository, and is made available under the terms and conditions applicable to Open Access Policy Articles, as set forth at http:// nrs.harvard.edu/urn-3:HUL.InstRepos:dash.current.terms-of-use\#OAP

\section{Share Your Story}

The Harvard community has made this article openly available.

Please share how this access benefits you. Submit a story.

Accessibility 


\section{Maximizing The Energy Density of Dielectric Elastomer Generators Using Equi-Biaxial Loading}

By Jiangshui Huang*, Samuel Shian*, and Zhigang Suo†, David R. Clarke $\dagger$

[*] These authors contributed equally to this work.

[†]Prof. Z. Suo, Prof. D. R. Clarke, Dr. J. Huang, Dr. S. Shian

School of Engineering and Applied Sciences, Harvard University, Cambridge, MA 02138 (USA)

E-mail: suo@seas.harvard.edu, clarke@seas.harvard.edu

Keywords: Dielectric Elastomer Generator, Electromechanical, Capacitance, Viscosity, Energy

Dielectric elastomer generators (DEGs) for harvesting electrical energy from mechanical work have been demonstrated but the energy densities achieved are still small compared with theoretical predictions. We show that significant improvements in energy density $(560 \mathrm{~J} / \mathrm{kg}$ with a power density of $280 \mathrm{~W} / \mathrm{kg}$ and an efficiency of $27 \%$ ) can be achieved using equi-biaxial stretching, a mechanical loading configuration that maximizes the capacitance changes. We demonstrate the capacitance of dielectric elastomers subjected to equi-biaxial stretches is proportional to the fourth power of the stretch. Quantification of the individual energy contributions indicates that attaining higher conversion efficiencies is limited by viscous losses within the acrylic elastomer, suggesting that still higher conversion efficiencies with other elastomers should be attainable with our novel mechanical loading design. 


\section{Introduction}

Dielectric elastomer generators (DEGs) are attractive for harvesting electrical energy from mechanical work because they comprise relatively few moving parts and because large elastomer sheets can be mass produced. Demonstrations of harvesting electrical energy from diverse mechanical energy sources, including ocean waves, wind, flowing water and human movement, $[1,2,3,4]$ have been reported. Also demonstrated has been the charging of small electrical devices such as cell phones and sensors embedded in human body for medical applications. ${ }^{[1,2,4]}$ Although various prototypes, using different materials and various harvesting circuits and cycles, have been reported in the literature ${ }^{[2,3-5,6,7,8]}$ since DEGs were first proposed in $2001^{[1]}$, the energy densities achieved to date are still far less than the theoretical prediction of $1700 \mathrm{~J} / \mathrm{kg}$ based on acrylic elastomers ${ }^{[9]}$. The energy density is defined as the net electrical energy harvested in one electromechanical cycle over the active mass of the elastomer. For example, an ocean wave energy harvesting buoy has an energy density of about $135 \mathrm{~J} / \mathrm{kg}$, a DEG with balloon geometry has an energy density of $102 \mathrm{~J} / \mathrm{kg}$, and self-priming DEGs have been reported with energy densities from $2.8 \mathrm{~J} / \mathrm{kg}$ to $12.6 \mathrm{~J} / \mathrm{kg} .[3,4,6,7,8]$. A relatively high energy density, $300 \mathrm{~J} / \mathrm{kg}$, has been reported for shoe generators ${ }^{[8]}$, but the significance of this value is difficult to evaluate because only limited technical details were reported. While most of the above harvesting systems were designed to operate over a long lifetime which might have lowered the attainable energy density to a certain extent, we are focusing on maximizing the energy density by imposing loading conditions probably close to the electrical and mechanical limits of the elastomer, without explicitly considering the lifetime 
of the DEG. As we will show, considerably higher energy densities can be achieved using equi-biaxial mechanical loading since this configuration maximizes the capacitance changes and hence the electrical energy generated in an electromechanical cycle.

A DEG is, in essence, a mechano-capacitive device creating electrical energy by transferring charge from a low to high voltage. Since the capacitance of a dielectric elastomer (DE) sheet increases as it is stretched in area and squeezed in thickness, the achievable energy density depends on how much the capacitance can be changed during an electromechanical cycle. This capacitance change, in turn, depends strongly on the loading geometry. It can be shown that uniaxial, pure-shear, and equi-biaxial stretching configurations increase the capacitance in proportion to the first, second and fourth power of the stretch, $\lambda$, respectively, where $\lambda$ is defined as the linear length ratio of the deformed and the un-deformed states in the direction of the stretching force. ${ }^{[10]}$

This fourth power dependence has not been exploited in previously reported DEG configurations. For example, for a DE sheet stretched over a pressurized hole, the state of stretch in the sheet is inhomogeneous, being equi-biaxial at the center of the sheet and pure-shear at the edge of the sheet.[7] Here we take advantage of the high capacitance changes afforded using equi-biaxial loading. An average energy density of $560 \mathrm{~J} / \mathrm{kg}$ has been achieved which, to the best of our knowledge, is the highest energy density yet reported At the same time, the average power density is $280 \mathrm{~W} / \mathrm{kg}$ and the average efficiency obtained is $27 \%$, within the first eight cycles of operation. Although this efficiency is several times higher than a recent report of an efficiency of $7.5 \%{ }^{[7]}$ using the same acrylic elastomer, we will show that the 
efficiency is mainly limited by the viscous losses in the acrylic elastomer, indicating that significantly higher efficiencies should be attainable with other elastomers.

\section{Experimental Section}

\subsection{Materials}

In common with many previous studies of DEGs, thin sheets of acrylic elastomers (VHB 4905, $3 \mathrm{M}$ ) were used as the dielectric and a layer of carbon conductive grease (MG Chemicals CAT. NO. 846-80G) was coated on both sides to serve as compliant electrodes. The elastomer thickness, $H$, was $0.5 \mathrm{~mm}$, and the electroded radius, $R_{0}$, was $2.0 \mathrm{~cm}$ corresponding to a generator mass of $M=0.60 \mathrm{~g}$.

\section{2 . Mechanical Loading Configuration}

The mechanical loading configuration was modified from that in our previous work on actuators $^{[11]}$. Equi-biaxial loading was accomplished by applying radial forces to the circumference of the DE sheet through an assembly of clips, wires $(\varnothing 0.23 \mathrm{~mm}, 1080$ carbon steel) and pulleys, all loaded by the motion of a linear servo-motor (Model SLP35, Nippon Pulse America Inc.), which is schematically shown in Figure 1a and $1 \mathrm{~b}$. The applied force, $F$, was recorded with an in-line load cell (Model LSB200, Futek). The instantaneous stretch of the DE sheet, defined as $\lambda=R / R_{0}$ ( $R$ is the radius of the sheet at the stretched state), as a function of the servo displacement, $L$, was determined from images recorded by a camera mounted over the DE sheet. In the experiments, the servo displacement ranged from o to 105 
$\mathrm{mm}$ in each electromechanical cycle, corresponding to a range of the radial stretch from 1.2 to 5.4. Two snap-shots of the DE sheet corresponding to the minimum and the maximum stretches are shown in Figure 1c and 1d, respectively.

\section{3 . Electric Harvesting Circuit}

The electrodes of the DE under cyclic mechanical load were connected to an electrical circuit for electric energy harvesting (Figure 2a). Charge at a low voltage $\left(\Phi_{L}\right)$ was delivered from a power supply (Trek, model 610E), and charge at a high voltage $\left(\Phi_{H}\right)$ was harvested by a large capacitor. The large capacitor implicated that the voltage of the capacitor remained constant throughout the electromechanical cycles. The diode $D_{1}$ (NTE517, NTE Electronics, Inc) only allowed the charging current to flow when the voltage across the DE, $\Phi_{D E}$, was lower than that of the power supply, $\Phi_{L}$. The diode $D_{2}$ only allowed the charge to flow from the DE to the harvesting capacitor when $\Phi_{D E}>\Phi_{H}$. Thus, the diodes $D_{1}$ and $D_{2}$ acted as switches, turning on and off automatically depending on the voltage of the DE. Essentially, $\Phi_{D E}$ was varied between two fixed voltages, $\Phi_{L}$ and $\Phi_{H}$, as the DE sheet was cyclically stretched.

To illustrate the principle of the DEG presented in this paper, and for direct comparison with our observations, an idealized energy harvesting cycle is schematically shown on the voltage-stretch plane and the voltage-charge plane (Figure 2b). The schematics neglect dissipative processes ${ }^{[12]}$, and the electric energy consumed by the measurement sub-assemblies. Starting in the state A, where the DE is at a condition of minimal stretch, $\lambda_{\min }$, high voltage, 
$\Phi_{H}$, and low charge, $Q_{\text {low }}$, the DE is stretched under open-circuit conditions to the state $B$. In doing so, the charge remains constant and consequently the DE voltage decreases to that of the power supply, $\Phi_{L}$. At the state $B$, the power supply is connected and the stretching is continued to a pre-determined maximum stretch, $\lambda_{\max }$, at state C. During this step, charge flows from the power supply to the DE electrodes at a constant voltage of $\Phi_{L}$. When the maximum stretch is reached, state $\mathrm{C}$, the $\mathrm{DE}$ is at its highest charge $Q_{\text {high }}$. The power supply is then disconnected, and the stretching forces are decreased allowing the DE to shrink back elastically. As it does so, the DE capacitance decreases and since the charge remains constant, the voltage increases. At the state $\mathrm{D}$, the $\mathrm{DE}$ reaches a high voltage, $\Phi_{H}$, and is connected to the harvesting capacitor. $\Phi_{H}$ is chosen to be lower than the breakdown voltage of the DE. As the stretch further decreases, the charge on DE flows to the harvesting capacitor, until the DE has shrunk back to its minimally stretched state at the state A. So, in one complete cycle, a charge, $\Delta Q=Q_{\text {high }}-Q_{\text {low }}$, is boosted from the low voltage $\Phi_{L}$ to the high voltage $\Phi_{H}$, by converting part of the elastic energy stored in the DE. Correspondingly, the net electric energy harvested, $\Delta E_{N e t}$, is equal to $\Delta Q\left(\Phi_{H}-\Phi_{L}\right)$. The nonlinearity of the curves $A B$ and $C D$ in the voltage-stretch plane results from the nonlinear dependence of the capacitance on the stretch.

The complete circuit, including the measurement components, is shown in Figure 3. The charging current $i_{\text {charge }}$ is recorded with a voltmeter $V_{1}$ (model USB-6218, National Instruments) in parallel with a resistor $R_{1}, i_{\text {charge }}=\Phi_{1} / R_{1}$. Similarly, the harvesting current $i_{\text {harvest }}$ is recorded with a voltmeter $V_{2}$ in parallel with a resistor $R_{2}, i_{\text {harvest }}=\Phi_{2} / R_{2}$. The voltage across 
the DE, $\Phi_{D E}$, is measured using a voltmeter, $V_{3}$ through a voltage divider, $R_{3}$ and $R_{4}$, $\Phi_{D E}=\Phi_{3}\left(1+R_{4} / R_{3}\right)$. Ideally, to harvest the electric energy at a constant voltage, the capacitance of the capacitor should be infinitely large compared with the capacitance of the DE (Figure 2). Instead, we use a Zener diode assembly. The Zener voltage (reverse bias breakdown) corresponds to the harvesting voltage, and thus, on determining the qualities of the DEG such as energy densities and efficiencies, the diodes behaves as an infinitely large capacitor. The Zener diode assembly consists of fifty Zener diodes, (part no. 1N5271BDO35, Microsemi Commercial Components Group) each having a reverse bias breakdown voltage of $100 \mathrm{~V}$, connected in series to establish a constant harvesting voltage of $\Phi_{H}=5 \mathrm{kV}$ (Supporting Information, Figure S1).

\section{Results and Discussion}

The response of the equi-biaxial generator for the second to the fifth electromechanical cycles is shown in Figure 4 at a frequency, an input voltage, and a harvesting voltage of $0.5 \mathrm{~Hz}$, $2 \mathrm{kV}$, and $5 \mathrm{kV}$, respectively. These parameters, including the maximum stretch, are experimentally chosen to maximize the energy density while preventing premature failures, such as rupture, loss of tension, or electrical breakdown. For example, too high a harvesting voltage results in too high a Maxwell stress which delays the recovery of the acrylic dielectric elastomer in the relaxing process leading to the loss of tension of dielectric elastomers, and may even lead to electric breakdown. ${ }^{[13]}$ The harvesting cycles have the same basic characteristics as the ideal schematic shown in Figure 2b. The difference is the data, 
highlighted by the dashed rectangle in Figure 4a, which was attributed to the viscous response of the DE: When the servo moved back to the zero displacement, the DE did not recover the minimally stretched state immediately and thus the DE was nearly at a state of loss of tension within a small servo displacement. This effect of the viscosity of the DE is also evident in the plots of force, voltage and harvesting current when the servo displacement is prescribed as a function of time for the electro-mechanical cycles (Figure 5). First, as the cycle number increases, the maximum applied force decreases very fast initially and rather slowly after a few cycles. Second, there is a delay between the voltage shut-off and the beginning of the next loading cycle (mark A in Figure 5). Third, there is also a delay between the harvesting current shut-off and the beginning of the next loading cycle (mark B in Figure 5).

Charge was drawn from power supply to $\mathrm{DE}$ when the $\mathrm{DE}$ was stretched at the low voltage and charge from the DE was harvested when the DE was relaxed at the high voltage, as shown in Figure 5 marked by dashed lines $C$ and $D$, respectively. The shapes of the curves of the charging and the harvesting currents as a function of time result from the quartic dependence of the capacitance on the stretch. The charge drawn from the power supply in each cycle, $\Delta Q_{i n}$, was determined by integrating the charging current as a function of time, $\Delta Q_{i n}=\int_{T 0}^{T+T 0} i_{\text {charge }} d t$, and the harvested charge in each cycle, $\Delta Q_{h}$, was determined by integrating the harvesting current as a function of time, $\Delta Q_{h}=\int_{T 0}^{T+T 0} i_{\text {harvest }} d t$, where $T o$ and $T$ are the starting time of a cycle and the period of a cycle, respectively. $\Delta Q_{i n}$ and $\Delta Q_{h}$ for each cycle for the first nine cycles are shown in Figure 6a. Except the ninth cycle, where the charge drawn from the power supply was largely dissipated within the membrane thickness, most of the charge drawn from 
power supply was boosted up to a high voltage and then delivered to the harvesting capacitor in each cycle. The differences between $\Delta Q_{i n}$ and $\Delta Q_{h}$ for the first few cycles were a direct consequence of the mechanical viscous creep of the elastomer under load: The minimum stretches increases after the cycles and thus a fraction of charge remained on the DE (Supporting Information, Figure S2). The charge consumed by the voltage-measuring resistances $R_{3}$ and $R_{4}$ in each cycle, $\Delta Q_{R}$, determined as $\Delta Q_{R}=\int_{T 0}^{T+T 0} \Phi_{D E}(t) /\left(R_{3}+R_{4}\right) d t$, was less than $1 \%$ of the charge drawn from the power supply, $\Delta Q_{i n}$, so this part of energy harvested was neglected in determining the electric energy harvested (Supporting Information, Figure S3). Thus, the net electric energy harvested in each cycle, $\Delta E_{N e t}=\Delta Q_{h} \Phi_{H}-\Delta Q_{i n} \Phi_{L}$, and the energy density achieved is $E_{\text {Density }}=\Delta E_{\text {Net }} / M$, where $M$ is the mass of the electroded part of the elastomer. The average energy density for the first eight cycles is $560 \mathrm{~J} / \mathrm{kg}$, corresponding to an average power density $280 \mathrm{~W} / \mathrm{kg}$. After the first eight electromechanical cycles, the $\mathrm{DE}$ fails to relax to a small stretch under electric force and stays in a loss-of-tension state at a rather high stretch, which may be due to creep of the acrylic elastomer, and thus the DEG failed to harvest electrical energy effectively.

As the servo displacement, $L$, was cyclically increased and decreased, the mechanical energy consumed in each cycle was determined by integrating the load-displacement cycle: $\oint F d L$ (Figure 7). The electromechanical and the pure mechanical cycles are cycles with and without electric charge on the $\mathrm{DE}$, respectively. For the purely mechanical cycle, the mechanical energy dissipated is mainly due to the viscous deformation of the elastomer while a small percentage of the energy is dissipated through the plastic deformation and the frictions 
of the steel wire and pulley arrangement (Supporting Information, Figure S4). The mechanical energy dissipated during an electromechanical cycle is denoted by $\triangle E_{E M C}$, and that for the purely mechanical cycle is denoted by $\Delta E_{P M C}$. Due to the relatively slow recovery time of the viscous creep of the elastomer, the mechanical energy dissipated per cycle decreased at increasing number of cycles (Figure 8a). The significant viscoelastic energy dissipation in the VHB elastomer material is evident when electromechanical and pure mechanical cycles are compared. $\triangle E_{E M C}$ and $\triangle E_{P M C}$ are comparable, demonstrating that a significant fraction of the mechanical energy was lost through viscous dissipation rather than being converted to electrical energy. Thus, the viscous losses of the mechanical energy in the acrylic elastomer limits the efficiency of the DEG, which is defined by the ratio of the net electric energy gained to the applied mechanical work: $\eta=\Delta E_{N e t} / \Delta E_{E M C}$. Nevertheless, the efficiency achieved is significantly higher than a recent report of an efficiency of $7.5 \%{ }^{[7]}$ using the same elastomer: The average efficiency of the first nine cycles is $27 \%$ (Figure $8 \mathrm{~b}$ ). This indicates that DEG's utilizing the biaxial mechanical loading configuration we have developed have the potential for significantly higher energy conversion efficiencies by replacing the VHB material with a more creep-resistant elastomer. An ideal elastomer would have the following additional characteristics: high stretch, high tear strength, high electrical breakdown strength, low leakage current, and long term stability [9].

To demonstrate the quartic dependence of the DE capacitance with respect to the stretch under equi-biaxial loading, we measured the amount of charge stored as a function of the stretch. The charge was delivered to the DE from a power supply with a pre-set voltage limit, 
$\Phi_{\text {preset }}=3 \mathrm{kV}$. The DE was first charged at a pre-set current limit until its voltage reached $\Phi_{\text {preset }}$

(Figure 9a). In this process, the voltages of the power supply and the DE increased at the same rate. Subsequently, the charging current was leakage current, which was far lower than the pre-set current limit, so the charge lost due to leakage could be neglected and thus the charge placed on the DE, $Q_{D E}$, corresponded to the shaded area (Figure 9a). Subjected to the electric field and the mechanical force applied, the stretch of the DE was measured directly using a camera mounted over the elastomer sheet. (The majority of the stretch was due to the mechanical force, and the electric field only slightly increased the stretch). $Q_{D E}$ was found to vary with the fourth power of the biaxial stretch as shown in Figure 9b, and the pre-set voltage limit, $\Phi_{\text {preset }}$, was constant, so the capacitance of the DE, $C_{D E}=Q_{D E} / \Phi_{\text {preset }}$, was indeed proportional to $\lambda^{4}$. Using the scaling obtained through simple geometric analysis, $C_{D E}=\left(\varepsilon_{0} \varepsilon \pi R_{0}^{2} / H\right) \lambda^{4}$, we determined the dielectric constant of the DE, $\varepsilon=4.03 \pm 0.17$, indicating the dielectric constant is independent of mechanical stretch.

\section{Conclusions}

By utilizing an equi-biaxial loading geometry we have shown that a DEG having significantly improved energy density, conversion efficiency, and power density can be achieved. The capacitance change is also consistent with the expected quartic scaling, $C_{D E} \propto \lambda^{4}$, under equi-biaxial loading rather than lower exponents for other mechanical loading configurations. These results provide fundamental understanding of the importance of electromechanical loading in the design of DEGs for electrical energy harvesting. 


\section{Supporting Information}

Supporting Information is available from the Wiley Online Library or from the author.

\section{Acknowledgements}

Our research was supported by the Harvard MRSEC program of the National Science Foundation (DMR-0820484) and by Army Research Office (W911NF-09-1-0476). The authors are grateful to Tongqing Lu for discussions.

Received: ((will be filled in by the editorial staff))

Revised: ((will be filled in by the editorial staff))

Published online: ((will be filled in by the editorial staff))

[1] R. Pelrine, R. Kornbluh, J. Eckerle, P. Jeuck, S. J. Oh, Q. B. Pei, S. Stanford, P Soc Photo-Opt Ins 2001, 4329, 148.

[2] S. Ashley, Sci Am 2003, 289, 52; C. Jean-Mistral, S. Basrour, J. J. Chaillout, Proc Spie 2008, 6927; S. Chiba, M. Waki, in Recent Advances in Wireless Communications and Networks, (Ed: J.-C. Lin), InTech, Rijeka, Croatia 2011, 435; S. Chiba, M. Waki, R. Kornbluh, R. Pelrine, Smart Mater Struct 2011, 20.

[3] S. Chiba, M. Waki, R. Kornbluh, R. Pelnine, Proc Spie 2008, 6927.

[4] R. D. Kornbluh, R. Pelrine, H. Prahlad, A. Wong-Foy, B. McCoy, S. Kim, J. Eckerle, T. Low, Mrs Bull 2012, 37, 246.

[5] C. M. Ihlefeld, Z. H. Qu, Proc Spie 2008, 6927; Y. H. Iskandarani, R. W. Jones, E. Villumsen, Proc. of SPIE, EAPAD 2009, 7287, 72871Y; C. Graf, J. Maas, D. Schapeler, in Electroactive Polymer Actuators and Devices, Vol. 7642 (Ed: Y. BarCohen), Spie-Int Soc Optical Engineering, Bellingham 2010.

[6] T. Mckay, B. O'Brien, E. Calius, I. Anderson, Appl Phys Lett 2010, 97; T. Mckay, B. O'Brien, E. Calius, I. Anderson, Smart Mater Struct 2010, 19; T. G. McKay, B. M. O'Brien, E. P. Calius, I. A. Anderson, Appl Phys Lett 2011, 98.

[7] R. Kaltseis, C. Keplinger, R. Baumgartner, M. Kaltenbrunner, T. F. Li, P. Machler, R. Schwodiauer, Z. G. Suo, S. Bauer, Appl Phys Lett 2011, 99.

[8] R. D. Kornbluh, R. Pelrine, H. Prahlad, A. Wong-Foy, B. McCoy, S. Kim, J. Eckerle, T. Low, in Electroactive Polymer Actuators and Devices, Vol. 7976 (Eds: Y. BarCohen, F. Carpi), Spie-Int Soc Optical Engineering, Bellingham 2011.

[9] S. J. A. Koh, C. Keplinger, T. F. Li, S. Bauer, Z. G. Suo, Ieee-Asme T Mech 2011, 16, 33.

[10] T. Lu, Z. Suo, Acta Mechanica Sinica 2012, 28, 1106.

[11] J. S. Huang, T. F. Li, C. C. Foo, J. Zhu, D. R. Clarke, Z. G. Suo, Appl Phys Lett 2012, 100. 
[12]C. C. Foo, S. J. A. Koh, C. Keplinger, R. Kaltseis, S. Bauer, Z. G. Suo, J Appl Phys 2012, 111. [13]J. Huang, S. Shian, R. M. Diebold, Z. Suo, D. R. Clarke, Appl Phys Lett 2012, 101, 122905. 
(a)
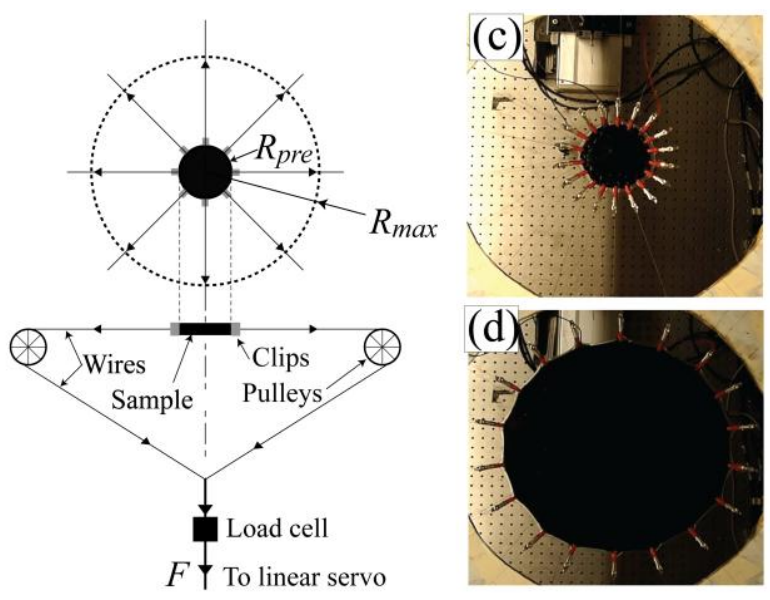

(b)

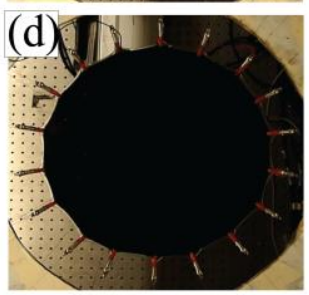

Figure 1. Schematic of the equi-biaxial mechanical loading configuration, (a) the top down view and (b) the side view. During an electromechanical cycle, the radius of dielectric elastomer sample changes from $R_{p r e}$ at a small prestretch to $R_{\max }$ at a pre-set maximum stretch by the pulling action of steel wires connected around the sample perimeter. The force, $F$, applied to stretch the dielectric elastomers is measured by an in-line load cell. Two top-down view snap-shots correspond to (c) the minimum and (d) the maximum stretches of 1.2 and 5.4, respectively.

(a)

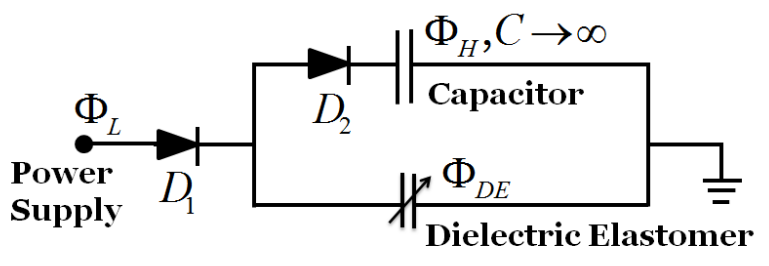

(b)
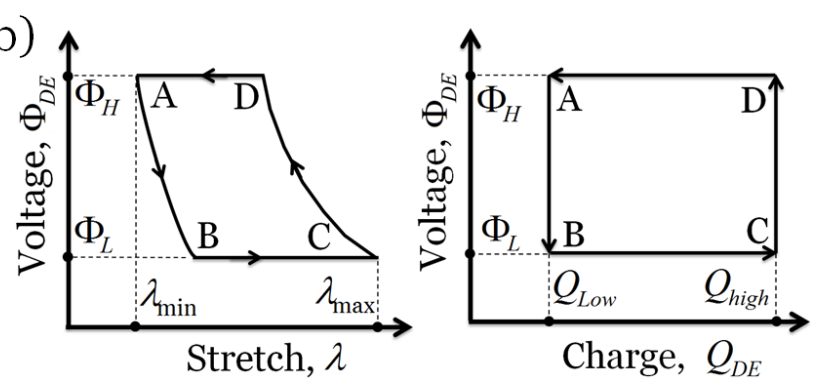

Figure 2 (a) Simplified electrical circuits for energy harvesting; (b) Simplified schematics show the principle of the DEG in an energy harvesting cycle. 


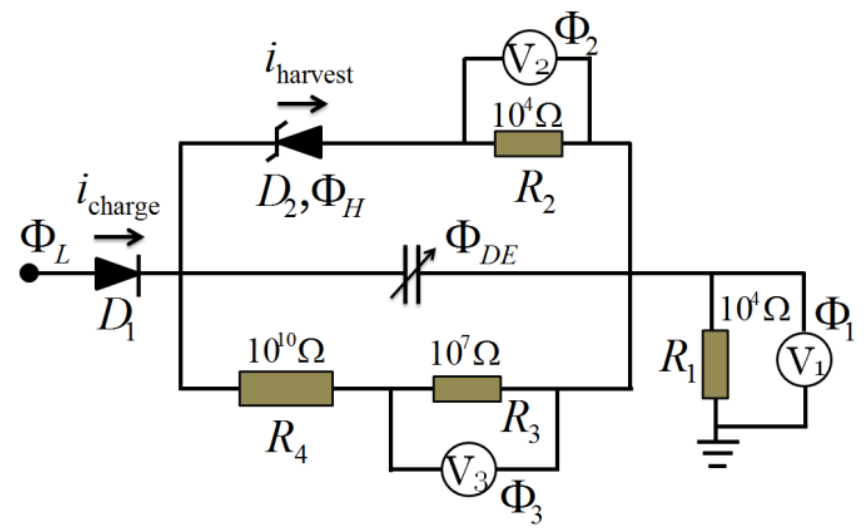

Figure 3 Electrical circuits including measuring circuits for energy harvesting. The reverse-biased Zener diode assembly, $D_{2}$, simulates an infinitely large harvesting capacitor.
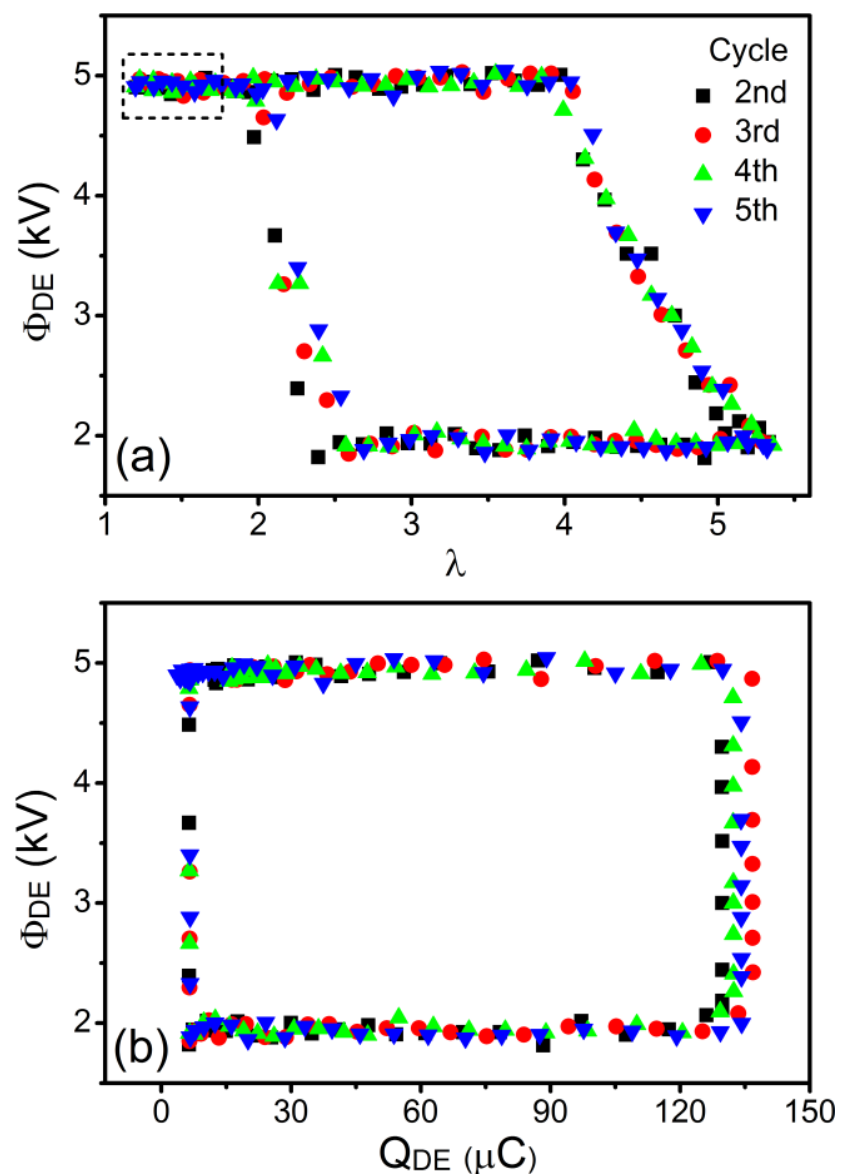

Figure 4. Voltage of the DE (a) as a function of the stretch and (b) as a function of the charge are shown from the second to the fifth cycles. 


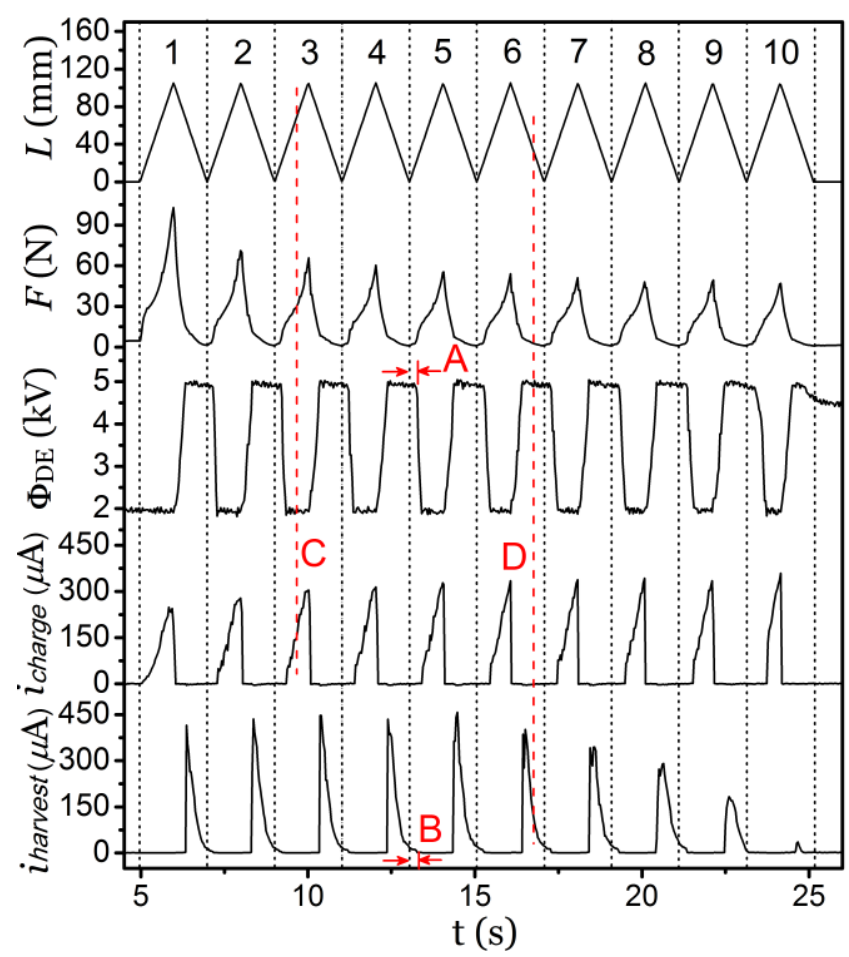

Figure 5 The servo displacement, $L$, the force applied to stretch the $\mathrm{DE}, F$, the voltage of the $\mathrm{DE}, \Phi_{D E}$, the charging electric current, $i_{\text {charge }}$, and the harvesting electric current, $i_{\text {harvest }}$, as a function of time, $t$. Cycle numbers , 1 to 10, are shown on the top.

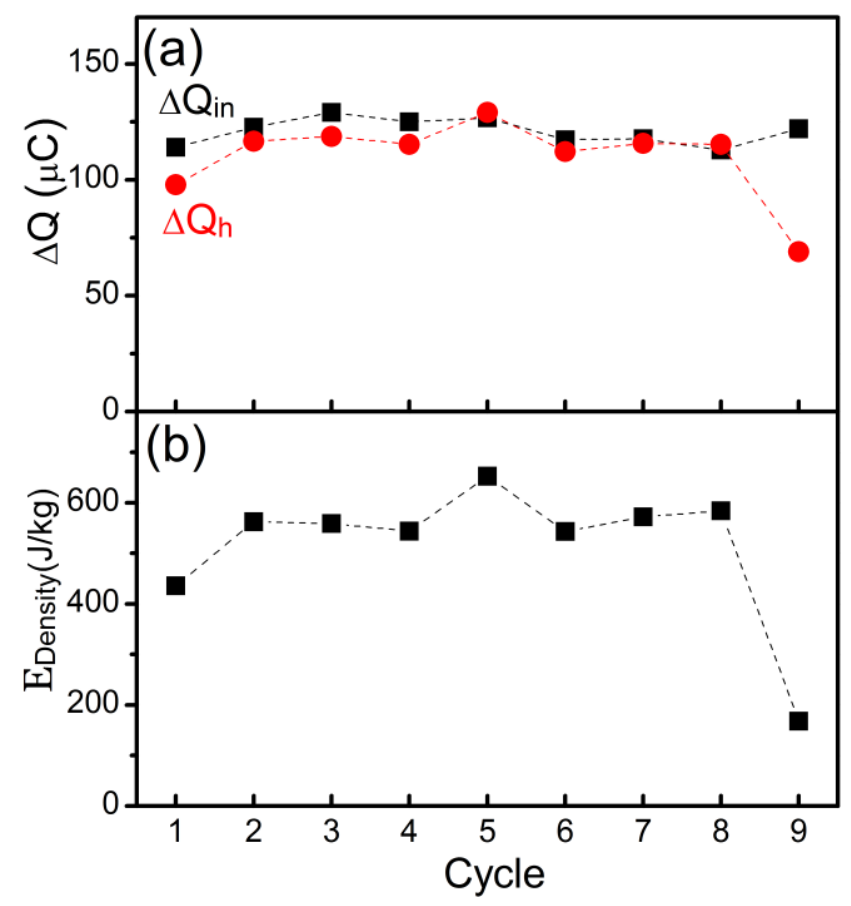


Figure 6 (a) The input charge drawn from the power supply, $\Delta Q_{i n}$, and the harvested charge, $\Delta Q_{h}$, in each cycle, corresponding to the cycle number shown in Figure 5.

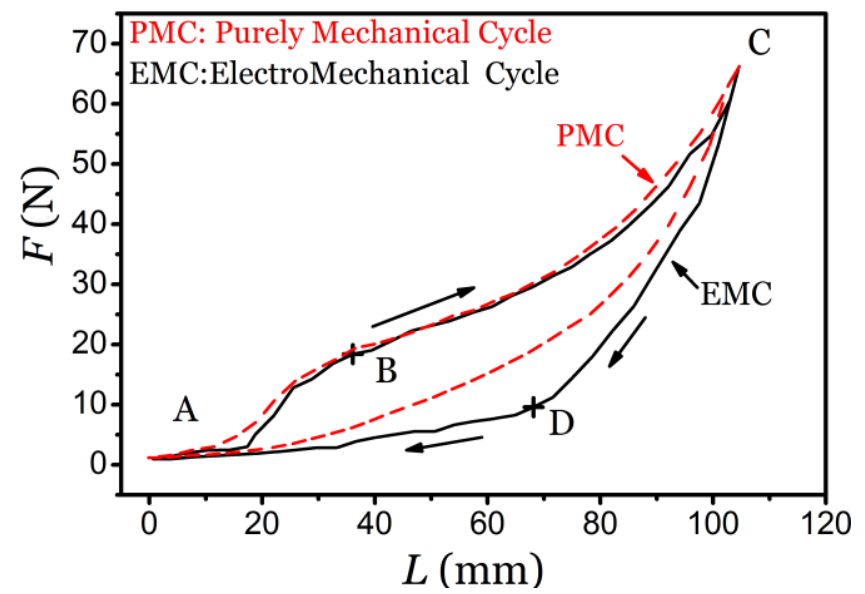

Figure 7 The force, $F$, as a function of servo displacement, $L$, for an electromechanical cycle (EMC) and a purely mechanical cycle (PMC, no charge was placed on the dielectric elastomer). The area enclosed by each curve corresponds to the mechanical energy consumed during the cycle. Points A, B, C, and D correspond to the electromechanical state shown in Figure 2b. Both curves are the third cycle of a freshly prepared sample.

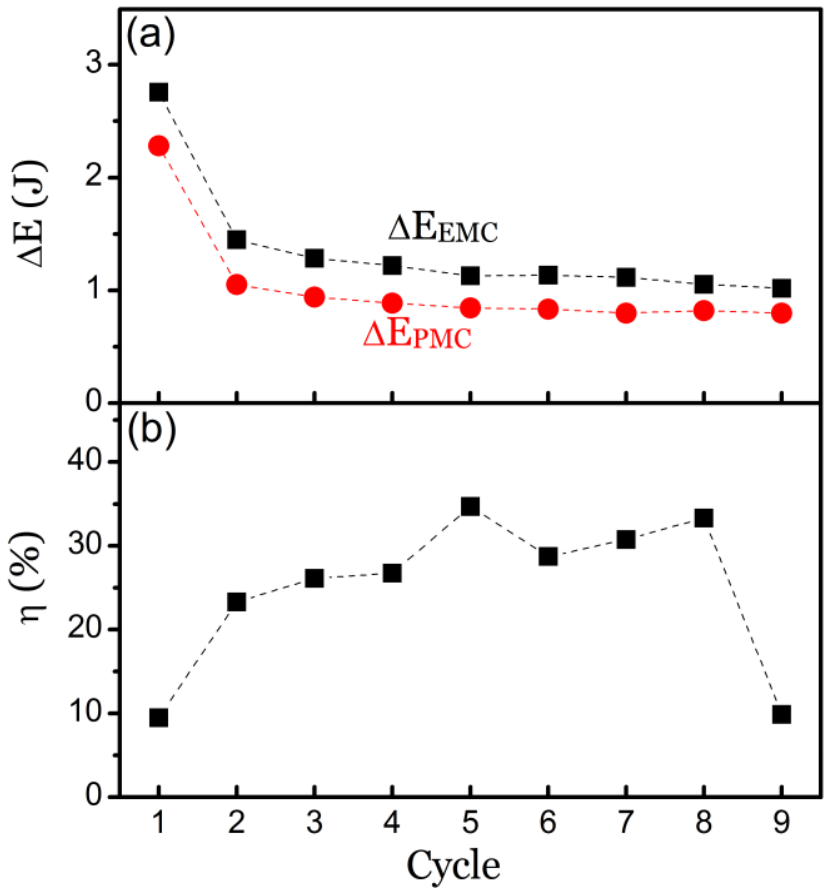

Figure 8 (a) The mechanical energy dissipated in an electromechanical cycle, $\Delta E_{E M C}$, and in a purely 
mechanical cycle, $\Delta E_{P M C}$, for the first nine cycles. The electromechanical cycles correspond to the cycles shown in Figure 5. (b) Energy conversion efficiencies in the electromechanical cycles.
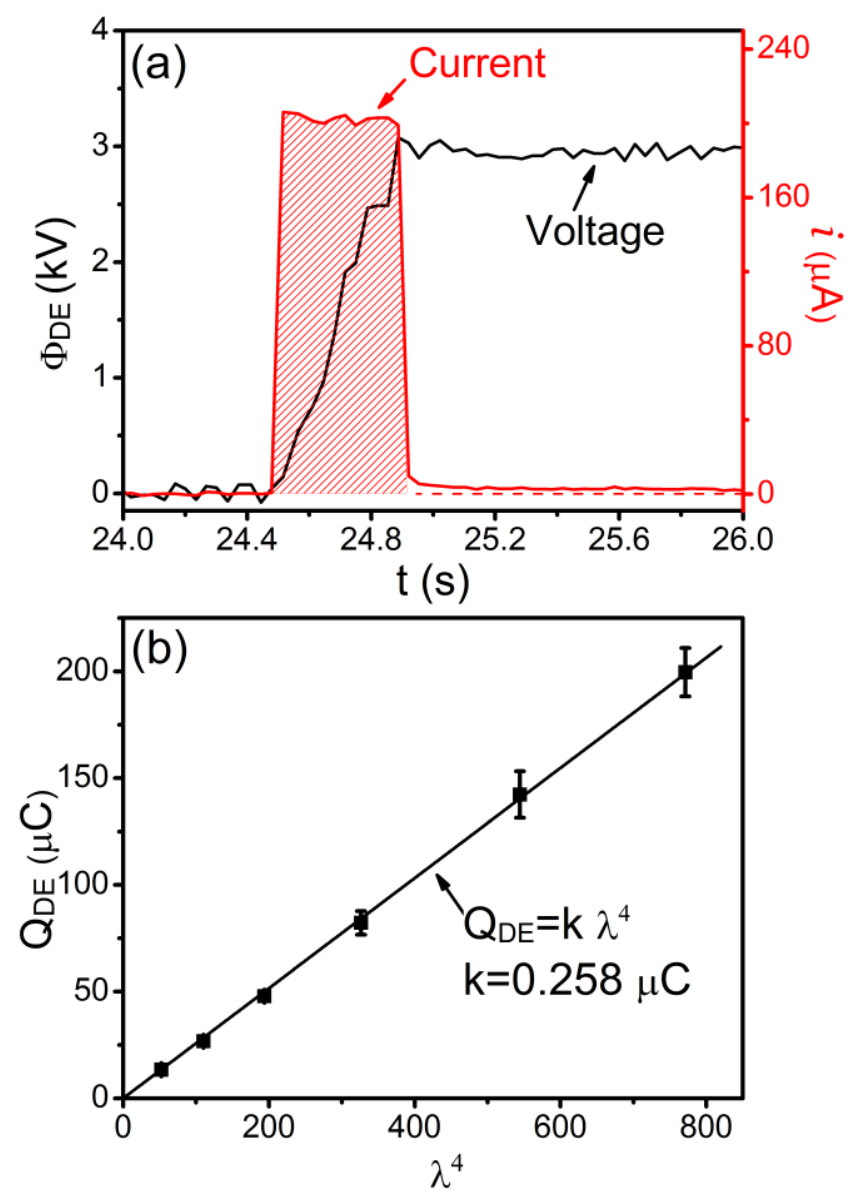

Figure 9. Demonstration of the quartic dependence of the DE capacitance with respect to the stretch by measuring the charge stored at various stretches. (a) An example of the charging measurements at a fixed stretch of 4.25 and at a current limit of $200 \mu \mathrm{A}$; the voltage of the dielectric elastomer, $\Phi_{D E}$, and charging current, $i$, are shown as a function of time. At each stretch, the current limit was chosen in such a way so that the magnitude of the leakage current was negligible at the maximum voltage and that the sampling time was adequate for voltage and current measurements; In general, the higher the stretch, the higher the current limit. The charge stored on the dielectric elastomer, $Q_{D E}$, corresponded to the shaded area. (b) The charge placed on the dielectric elastomer varies well with the fourth power of stretch: $Q_{D E}=k \lambda^{4}$, where $k=0.258 \mu C$. Since the voltage was constant at $3 \mathrm{kV}$, the DE capacitance varies with the fourth power of stretch as well. 


\section{Supporting Information}

for

\section{Maximizing The Energy Density of Dielectric Elastomer Generators Using Equi-Biaxial Loading}

By Jiangshui Huang*, Samuel Shian*, Zhigang Suo $\dagger$, David R. Clarke $\uparrow$

School of Engineering and Applied Sciences, Harvard University, Cambridge, MA 02138

[*]These authors contributed equally to this work.

[†]E-mail: suo@seas.harvard.edu, clarke@seas.harvard.edu

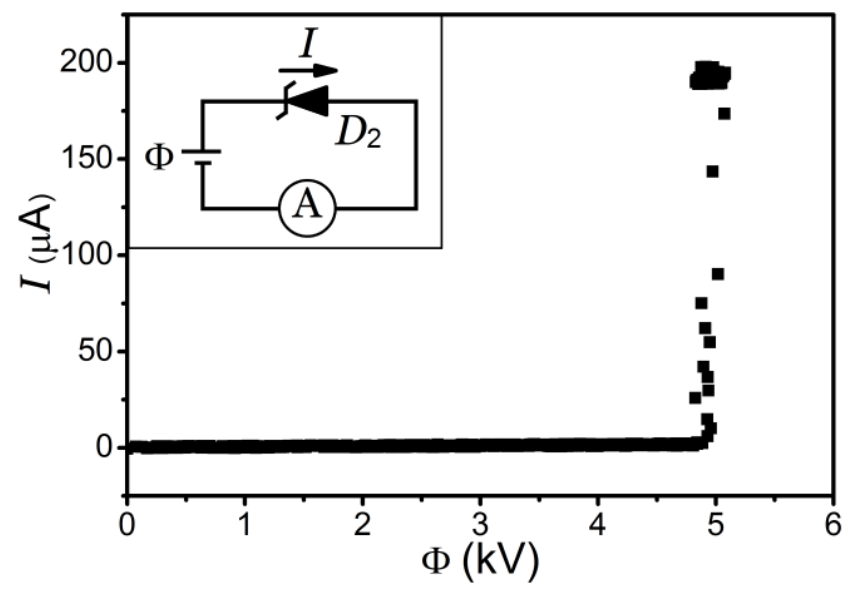

Figure S1. The electrical characteristics of the Zener diode assembly $D_{2}$ under reverse bias conditions showing that current only flows when the voltage exceeds $5 \mathrm{kV}$. This assembly is used as a capacitor to harvest current when the voltage on the DEG exceeds the reverse bias breakdown voltage. The current limit of the power supply was set at $200 \mu \mathrm{A}$. The insert at up left corner is the testing circuit. 


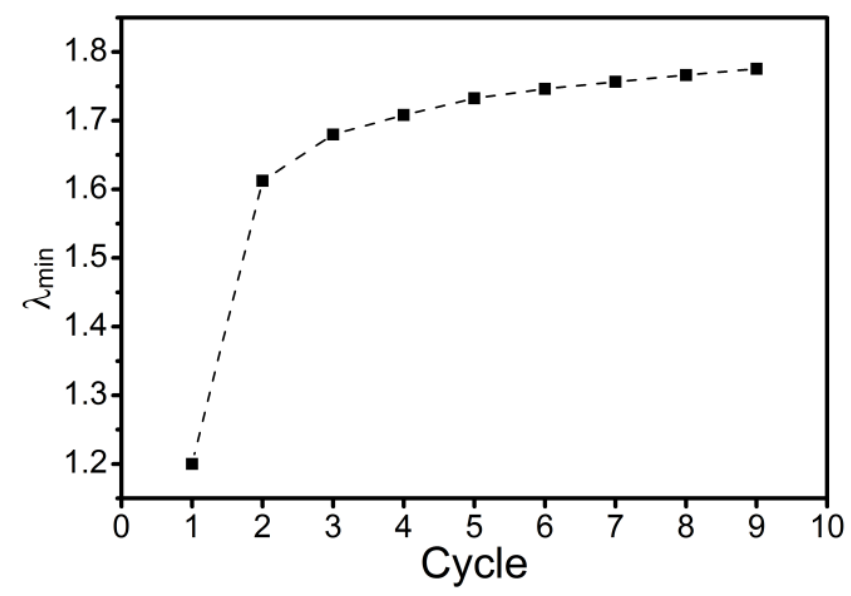

Figure S2. The minimum stretch, $\lambda_{\min }$, increases as the number of cycle increase, which is caused by the viscoelasticity of the elastomer.

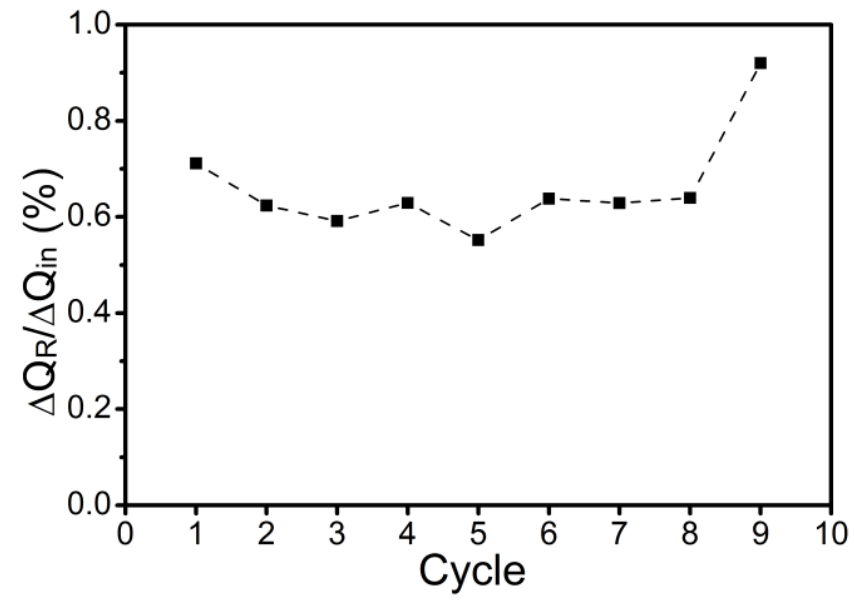

Figure S3. The charge delivered to the resistors $R_{3}$ and $R_{4}, \Delta Q_{R}$, is less than $1 \%$ of the charge drawn from power source, $\Delta Q_{i n}$, in each cycle, which indicates that the charge loss by the voltage measuring circuit is negligible. 


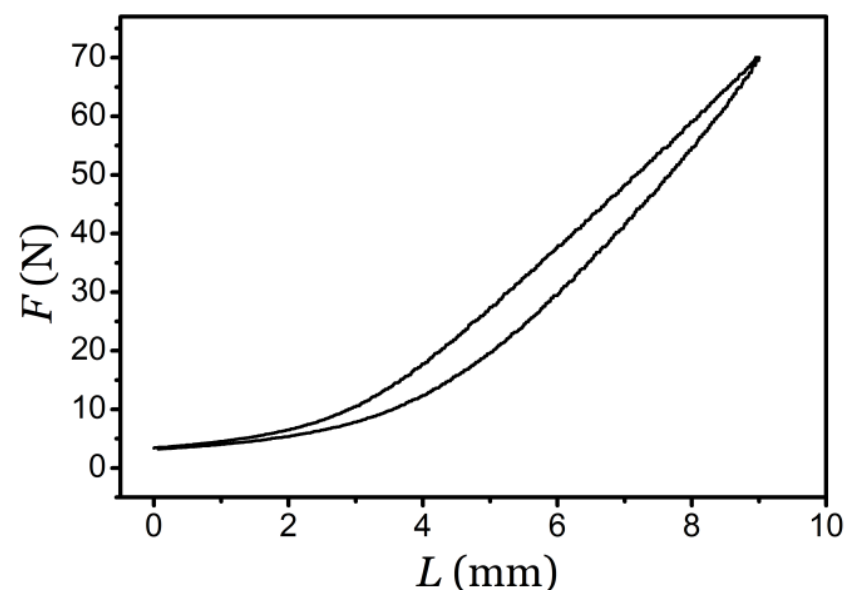

Figure S4. The mechanical energy dissipated in the mechanical loading system of steel wires and pulleys used to stretch the DE. The area under the curve is only about $4 \%$ of the mechanical energy dissipated in the purely mechanical cycle, $\Delta E_{P M C}$, shown in Figure 7. The data is recorded for the same maximum force and the same cycle frequency as the one used in the DEG experiment. 